\title{
Structure of an Oncology Information System Based on a Cost-Effective Relational Database for Small Departments of Radiation Oncology
}

 \\ Jiho $\mathrm{Nam}^{3 \text { [D }}$, Dong Hyeon $\mathrm{Kim}^{3 \text { [i] }}$ \\ 'Department of Radiation Oncology and Research Institute for Convergence of Biomedical Science and Technology, Pusan National \\ University Yangsan Hospital, ${ }^{2}$ Department of Radiation Oncology, Pusan National University School of Medicine, Yangsan, ${ }^{3}$ Department of \\ Radiation Oncology, Pusan National University Hospital, Busan, Korea
}

Received 19 October 2020 Revised 13 November 2020

Accepted 23 November 2020

\section{Corresponding author}

Yongkan Ki

(apex7171@hanmail.net)

Tel: 82-55-360-3450

Fax: 82-55-360-3449
Purpose: Radiation oncology information systems (ROIS) have evolved toward connecting and integrating information between radiation treatment procedures. ROIS can play an important role in utilizing modern radiotherapy techniques that have high complexity and require a large amount of information.

Methods: Using Access ${ }^{\top M}$ software, we have developed a relational database that is highly optimized for a radiotherapeutic workflow.

Results: The prescription table was chosen as the core table to which the other tables were connected, and three types of forms-charts, worklists, and calendars- were suggested. A fast and reliable channel for delivering orders and remarks according to changes in the situation was also designed.

Conclusions: We expect our ROIS design to inspire those who need to develop and manage an individual ROIS suitable for their radiation oncology departments at a low cost.

Keywords: Radiation oncology information system, Relational database, Microsoft access

\section{Introduction}

Since the awareness of the need for electronic recording and verification of radiotherapy treatments arose over 40 years ago [1-3], radiation oncology information systems (ROIS) have evolved toward connecting and integrating information between different radiation treatment procedures [4]. ROIS can play an important role in utilizing modern radiotherapy techniques that have high complexity and involve a large amount of information because it is essential to share such information quickly and efficiently among medical staff. Han et al. [5] reported that the overall workload associated with radiation treatment procedures such as nursing, simulation, and treatment was reduced by $28.2 \%$ through the introduction of an electronic chart system.

One of the most widely used digital database formats is the relational database (RDB) proposed by Codd [6] in 1970 . Since the RDB can effectively and intuitively manage a large amount of complex data using related tables and support data consistency and concurrency, this method is suitable for ROIS, which requires quick and accurate propagation of frequently updated treatment-related information. Although commercial radiotherapy platforms such as Aria ${ }^{\mathrm{TM}}$ 
(Varian Medical Systems, Palo Alto, CA, USA) or Mosaiq ${ }^{\mathrm{TM}}$ (Elekta Oncology Systems, Crawley, UK) already provide most ROIS functionality, their structures or functions cannot be rapidly customized to the needs of individual users, and the number of terminals in these platforms is limited. Therefore, they have limited capability to respond to the detailed requirements of individual departments.

This study aimed to suggest ROIS with an RDB structure that is effective for small radiation oncology departments using the cost-effective popular database software Access ${ }^{\mathrm{TM}}$ (Microsoft, Redmond, WA, USA). Since the feasibility of ROIS developed using this general-purpose software has already been shown by Lim et al. [7], in this study, we focus on an RDB structure that is more sophisticated and optimized for radiotherapeutic workflow. The treatment parameters directly related to treatment-beam delivery, such as the monitor unit, gantry angle, and source-to-surface distance, are excluded from our ROIS because they are effectively displayed by existing treatment machine-operating platforms.

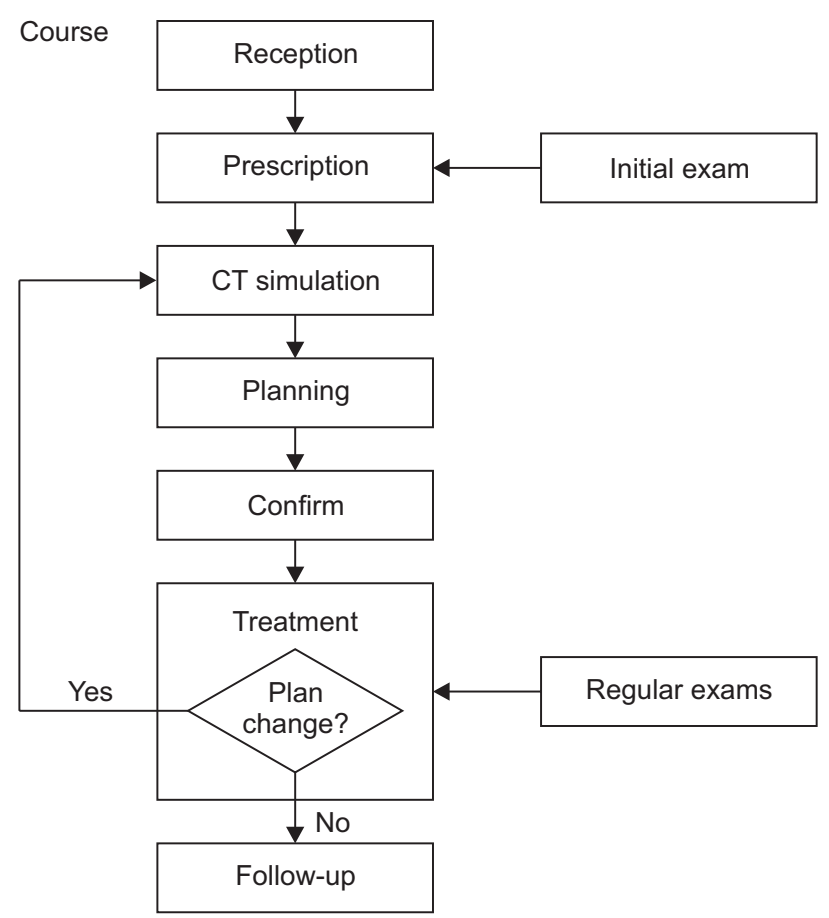

Fig. 1. Overall workflow for radiation treatments. CT, computed tomography.

\section{Materials and Methods}

The workflow of our department from the reception of patients to follow-up is presented as an example in Fig. 1. The workflow was carefully analyzed for the design of our own ROIS structure.

Using Access ${ }^{\mathrm{TM}}$, we created eight relational tables containing their own data and designed three types of forms for displaying the queried data. Detailed explanations are provided in the rest of this section. We established three design requirements to build ROIS that were optimized for our departmental workflow as follows.

\section{Core table}

Because all clinical procedures for radiation treatments should be implemented according to prescriptions, the prescription table was chosen as the core table. Moreover, a single course could be easily matched to a single prescription. Therefore, we added key information such as clinical status/findings and numerical prescriptions of treatment doses to the prescription table, as shown in Table 1. Five related tables were connected to the prescription table, and the rest were isolated. Fig. 2 presents a diagram describing the overall connections between the related tables.

\section{Change in situation}

The existing treatment plan may be changed frequently for various reasons, such as weight loss in a patient, modification of target lesions, and machine shutdown. During such situations, appropriate plan changes should be made as necessary to achieve the expected therapeutic effect. Moreover, these sudden changes should be quickly and accurately conveyed to all staff involved. Therefore, we designed order/remark tables to manage unexpected event information. The order table contains order information going from physicians to the other staff members, and the remark table contains information that should be shared between the staff members. When new order/remark content is fed into the ROIS, they are supposed to be immediately displayed on a related worklist to act as an alert. 
Table 1. Detailed contents contained in each table

\begin{tabular}{|c|c|}
\hline Table & Content \\
\hline Patient & $\begin{array}{l}\text { 1. Personal information (sex, age) } \\
\text { 2. Status (active, completed, suspended, canceled, etc.) } \\
\text { 3. Face photo link }\end{array}$ \\
\hline Prescription & $\begin{array}{l}\text { 1. Course number } \\
\text { 2. Referred information (clinic, physician, letter, etc.) } \\
\text { 3. Clinical status (stage, pathology, operation, chemotherapy, radiotherapy history, etc.) } \\
\text { 4. Clinical findings (lab, pathology, radiology, etc.) } \\
\text { 5. Prescription (physician name, aim, site, total dose/fraction, etc.) } \\
\text { 6. Setup photo link }\end{array}$ \\
\hline Plan & $\begin{array}{l}\text { 1. Binary (yes/no) values to indicate whether to complete the target, plan, and confirm } \\
\text { 2. Scheduled date and time for computed tomography simulation and treatment } \\
\text { 3. Assigned treatment room, machine, and technique names } \\
\text { 4. Dose/fraction per plan }\end{array}$ \\
\hline Exam & $\begin{array}{l}\text { 1. Scheduled exam date/time } \\
\text { 2. Status (initial, weekly, follow-up, etc.) } \\
\text { 3. Comments }\end{array}$ \\
\hline Order & $\begin{array}{l}\text { 1. Recorded date/time } \\
\text { 2. Content of order } \\
\text { 3. Binary (yes/no) value to indicate whether to implement }\end{array}$ \\
\hline Remark & $\begin{array}{l}\text { 1. Recorded date/time } \\
\text { 2. Content of remark } \\
\text { 3. Binary (yes/no) value to indicate whether to take care }\end{array}$ \\
\hline Shutdown & 1. Date to shutdown (weekend, holiday, etc.) \\
\hline User & $\begin{array}{l}\text { 1. User ID/password/name } \\
\text { 2. Working group }\end{array}$ \\
\hline
\end{tabular}

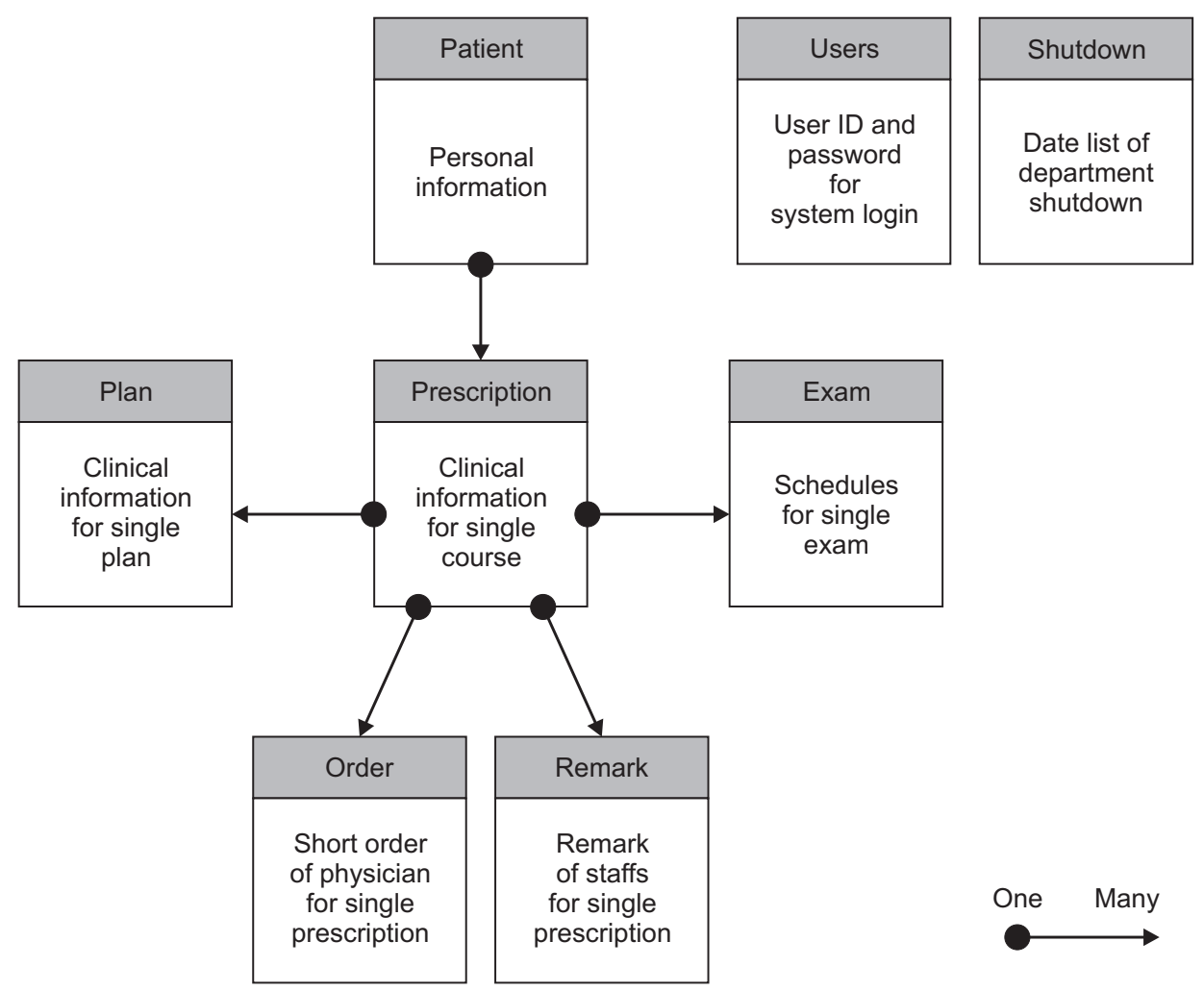

Fig. 2. Overall connections between the relational tables for radiation oncology information systems (ROIS). 


\section{Scheduling task}

Treatment scheduling is an inevitable task in radiotherapy departments because radiation treatment adopts a frac-

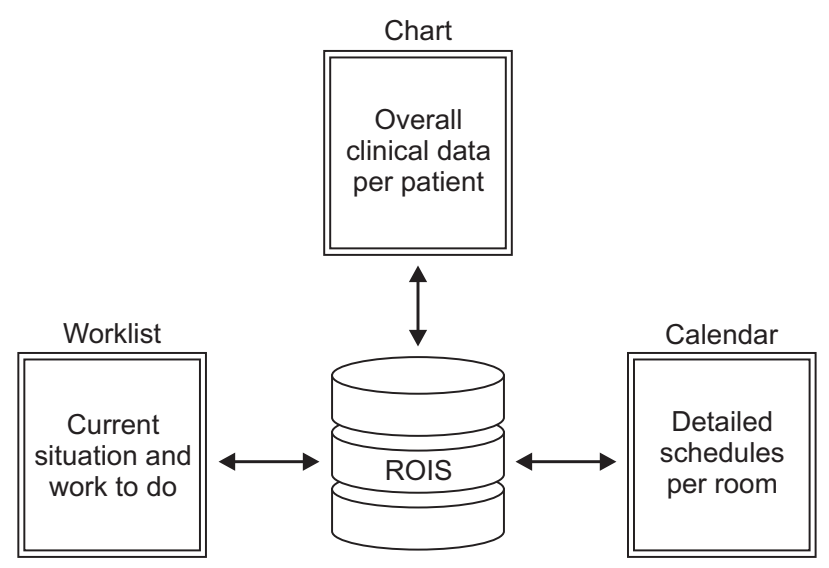

Fig. 3. Three kinds of forms for reviewing and editing radiation oncology information systems (ROIS) data.

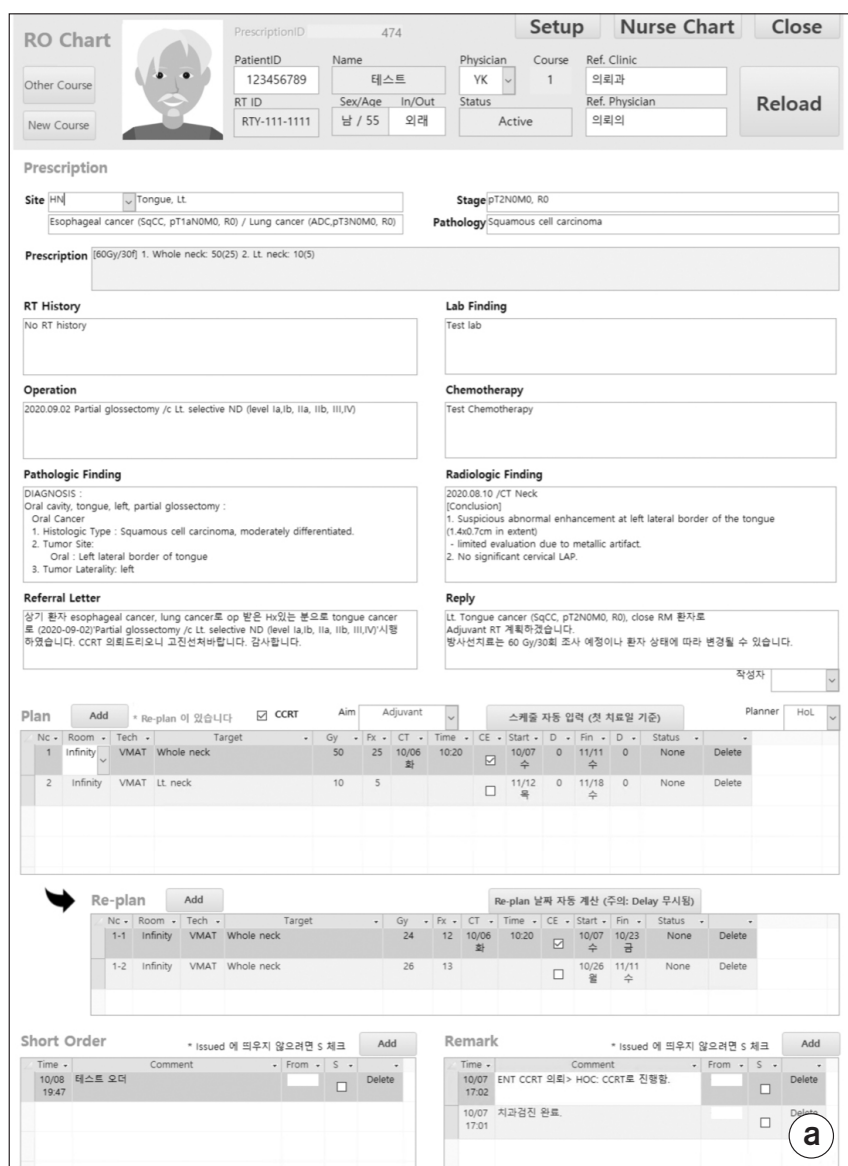

Fig. 4. (a) The main chart of a single patient, (b) the corresponding setup-photo viewer, and (c) the schedule chart for reception staff. 
treatment/exam schedules so that the information for each patient can be obtained at once. Additionally, the chart contains online addresses linked to a facial photo for personal identification, as well as CT simulation photos for checking the patient's posture, which are stored separately. The worklist form provides a list of the patient's information so that each staff member can easily plan what is to be done in each room. As mentioned in the previous paragraph, the latest orders and remarks are also displayed in the form of a worklist. The calendar form is provided for each room, which is similar to the worklist form. The worklist and calendar forms can help identify the tasks to be performed in each room.

\section{Results}

Fig. 4 shows the two chart forms designed in this study. One main chart contains most of the clinical data for a single patient, as shown in Fig. 4a, and the other chart is used by the reception staff to schedule examinations, as shown in Fig. 4c. The current clinical status and changes to the patient's treatment plan can be easily identified in short

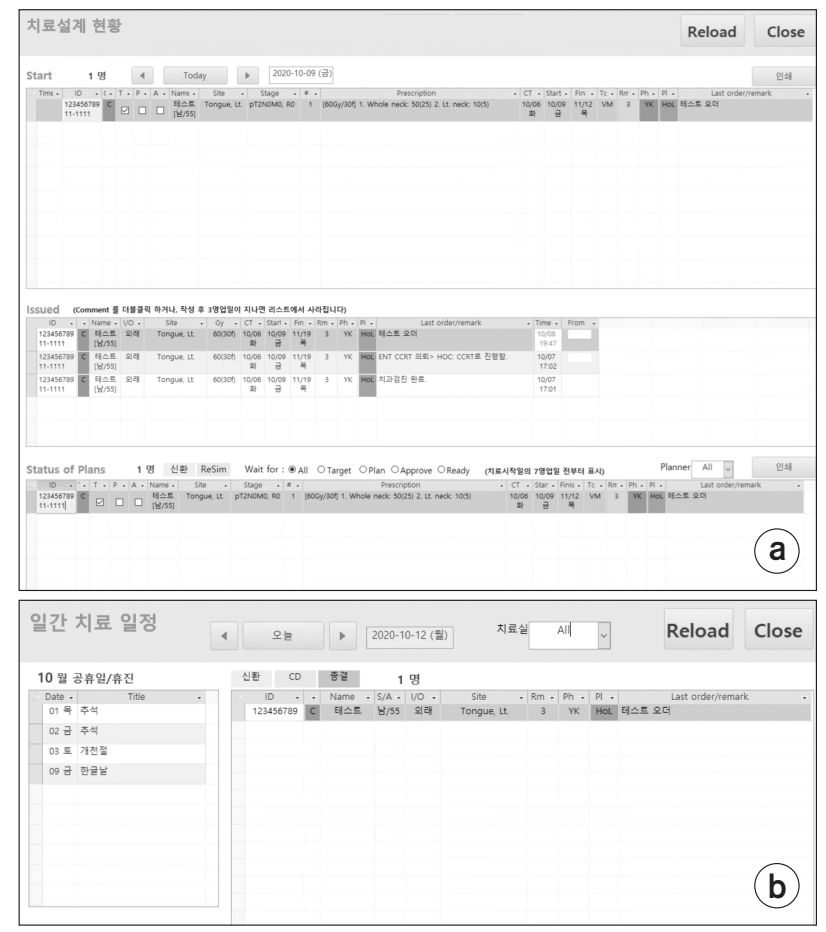

Fig. 5. Two worklist forms for (a) radiation treatment planning rooms and (b) treatment rooms as examples. order, and remarks are listed at the bottom of the chart. The chart also provides a viewing window to check the patient's posture during CT simulation, and the history of radiation treatment can be easily reviewed by clicking the corresponding course number.

Fig. 5 shows two worklist forms as examples. The worklist form for planners contains three sub-lists for the start of daily treatment, issued orders/remarks, and the current status of planning, as shown in Fig. 5a. The issued list can help planners to respond quickly to the reported situation, and the planners can easily track or conduct the radiation treatment plans of different patients based on the other two lists. The worklist form shown in Fig. 5b provides a daily treatment list for each treatment room, such that therapists can identify their own schedules. We designed five different worklist forms for physicians, planners, reception staff, CT simulation staff, and therapists. Fig. 6 shows two calendarstyle forms designed for displaying examination and CT simulation schedules. Since examination or treatment schedules may change frequently, a calendar form effective in managing the schedule information is necessary, especially for the reception staff. We noted that the worklist and calendar forms could offer complementary supports for identifying the task in each room.

In addition to the main functions described above, we in-



Fig. 6. Calendar-style forms for managing schedules of (a) exams and (b) computed tomography (CT) simulations. 
troduce a login procedure to manage user-specific authority for clinical data security and develop detailed search and statistics functions through various data-filtering processes.

\section{Discussion}

The possibility of communication errors among staff that may lead to clinical accidents has significantly decreased since the introduction of highly computerized ROIS databases. However, there is a possibility of communication errors in non-routine workflow based on sudden changes in the situation; in such cases, a fast and reliable channel for delivering information to related staff is essential. In this study, we developed ROIS that can exactly meet these requirements. All related staff can easily access additional short orders by physicians or sudden changes in the patient's condition.

The biggest advantage of our ROIS is that it can be fully customized according to the requirements of individual departments, because the difficulty and cost of database development and maintenance using Access ${ }^{\mathrm{TM}}$ are significantly lower than those of the existing database platforms. Therefore, users can achieve high work efficiency with a highly customized ROIS. For instance, the workload and probability of human errors can be minimized by automatic treatment scheduling (rather than manual writing).

The ROIS presented in this study have some limitations. First, Access ${ }^{\mathrm{TM}}$ has limited data storage (approximately 2 GB) and a small number of connected users. Thus, our ROIS are difficult to use for radiation oncology departments beyond a certain size. Second, we believe that our system cannot be inherently connected or integrated with existing commercial operating systems for radiation treatment. Instead, it can be used in parallel with systems to maximize work efficiency.

\section{Conclusions}

We expect our ROIS design to inspire those who need to develop and manage an individual ROIS suitable for their radiation oncology departments at low cost.

\section{Acknowledgements}

This research was supported by the Basic Science Research Program through the National Research Foundation of Korea (NRF) funded by the Korean government (MSIT) (2020R1C1C1013300).

\section{Conflicts of Interest}

The author has nothing to disclose.

\section{Availability of Data and Materials}

All relevant data are within the paper and its Supporting Information files.

\section{Author Contributions}

Conceptualization: Yongkan Ki, Wontaek Kim. Data curation: Hosang Jeon, Dong Woon Kim. Formal analysis: Hosang Jeon. Funding acquisition: Hosang Jeon. Investigation: Ji Hyeon Joo, Dong Hyeon Kim. Methodology: Dahl Park. Project administration: Dong Woon Kim. Resources: Yongkan Ki, Jiho Nam. Software: Hosang Jeon, Dong Woon Kim. Supervision: Yongkan Ki, Wontaek Kim. Validation: Hosang Jeon. Visualization: Hosang Jeon. Writing-original draft: Hosang Jeon. Writing-review \& editing: Yongkan Ki.

\section{References}

1. Rosenbloom ME, Killick LJ, Bentley RE. Verification and recording of radiotherapy treatments using a small computer. Br J Radiol. 1977;50:637-644.

2. Fredrickson DH, Karzmark CJ, Rust DC, Tuschman M. Experience with computer monitoring, verification and record keeping in radiotherapy procedures using a Clinac-4. Int J Radiat Oncol Biol Phys. 1979;5:415-418.

3. Morrey D, Smith CW, Belcher RA, Harding T, Sutherland WH. A microcomputer system for prescription, calculation, verification and recording of radiotherapy treatments. Br J Radiol. 1982;55:283-288.

4. Fong de Los Santos LE, Herman MG. Radiation oncology information systems and clinical practice compatibility: 
workflow evaluation and comprehensive assessment. Pract Radiat Oncol. 2012;2:e155-e164.

5. Han Y, Huh SJ, Ju SG, Ahn YC, Lim DH, Lee JE, et al. Impact of an electronic chart on the staff workload in a radiation oncology department. Jpn J Clin Oncol. 2005;35:470-474.
6. Codd EF. A relational model of data for large shared data banks. Commun ACM. 1970;13:377-387.

7. Lim S, Kim K, Ahn S, Lee SH, Lee R, Cho S. Database for patient information management in radiation oncology department. Prog Med Phys. 2018;29:23-28. 